\title{
Secondary Syphilis
}

National Cancer Institute

\section{Source}

National Cancer Institute. Secondary Syphilis. NCI Thesaurus. Code C128413.

The secondary stage of syphilis typically that is characterized by generalized rash

(including palms and soles), mucocutaneous lesions, and lymphadenopathy. It usually begins one to two months after the primary stage. 